\title{
Parameter depending almost monotonic functions and their applications to dimensions in metric measure spaces
}

\author{
Natasha Samko \\ (Communicated by Vladimir D. Stepanov)
}

2000 Mathematics Subject Classification. 26A48, 54C35, 26A16.

Keywords and phrases. Zygmund conditions, Bary-Stechkin class, indices of monotonic functions, metric measure space, local dimensions.

\footnotetext{
Abstract. In connection with application to various problems of operator theory, we study almost monotonic functions $w(x, r)$ depending on a parameter $x$ which runs a metric measure space $X$, and the so called index numbers $m(w, x), M(w, x)$ of such functions, and consider some generalized Zygmund, Bary, Lozinskii and Stechkin conditions. The main results contain necessary and sufficient conditions, in terms of lower and upper bounds of indices $m(w, x)$ and $M(w, x)$, for the uniform belongness of functions $w(\cdot, r)$ to Zygmund-BaryStechkin classes.

We give also applications to local dimensions in metric measure spaces and characterization of some integral inequalities involving radial weights and measures of balls in such spaces.
}

\section{Introduction}

Last decades there was observed an increasing interest to the study of function spaces whose characteristics may vary from point to point. A well known typical example is the generalized Lebesgue space $L^{p(\cdot)}$ with variable exponent, see for instance the surveying papers [2], [11], [25] on harmonic 
analysis in such spaces. Another example is a generalized Hölder space with a given dominant of continuity modulus, which may vary from point to point. Hölder spaces of variable order $\lambda(x)$, and more general variable generalized Hölder spaces, were studied in [9], [16], [28], [29], [30], [32], [31]. The generalized Hölder classes were there defined as spaces of functions whose continuity modulus at a point $x$ has a dominant (characteristic) $w(x, r)$, where $r \in[0, \ell], 0<\ell<\infty$ and $x$ runs a certain set $X \subseteq \mathbb{R}^{n}$ (typically an interval in $\mathbb{R}^{1}$, or either a domain or a unit sphere in $\mathbb{R}^{n}$ ). This characteristic belongs to this or other Zygmund-Bary-Stechkin class $\Phi_{\beta}^{\alpha}$ in the variable $r \in \mathbb{R}_{+}^{1}$ for every $x \in \mathbb{R}^{n}$ and one needs to have some properties of these functions uniform with respect to $x$.

But the main reason for this study lies in some problems in the theory of metric measure spaces with variable dimensions. As is known, the onedimensional Muckenhoupt condition (or the multi-dimensional one in the case of radial weights), includes integral constructions similar to those which are involved in the Zygmund integral condition. When such Muckenhoupt conditions are used within the frameworks of metric measure spaces $X$ where $\mu B(x, r)$ depends on the point $x$, one arrives at a similar problem of dependence of a function in the Zygmund-Bary-Stechkin class $\Phi_{\beta}^{\alpha}$ on a parameter $x$ running a metric measure space, see [12]. These facts led us to the study of Zygmund-Bary-Stechkin functions $w(x, r)$ depending on a parameter $x$ belonging in general to an arbitrary metric measure space $X$, which is undertaken in this paper.

The paper is organized as follows. In Section 2 we give necessary preliminaries. In Subsection (2.1), we deal with the so called indices $m(w, x)$ and $M(w, x)$ of functions $w(x, r)$ almost monotonic in $r \in \mathbb{R}_{+}^{1}$. We refer to papers [10], [18], [17], [21], [22], [20], [19], [24], [23] for properties of such indices in the case $w=w(r)$ and their usage in the study of the Fredholmness of singular integral operators in weighted generalized Hölder spaces (see also [15] for Fredholmness in the case of usual Hölder spaces). In Subsection (2.1), we consider the index numbers $m(w, x), M(w, x)$ of Zygmund-Bary-Stechkin functions in dependence on a point $x$. We study, in particular, their lower and upper bounds when $x$ runs $X$. In Subsections (2.3) and (2.4), we deal with some generalized Zygmund, Bary, Lozinskii and Stechkin conditions.

Section 3 contains results on the characterization of the uniform belongness of functions to Zygmund-Bary-Stechkin classes in terms of lower and upper bounds of indices $m(w, x)$ and $M(w, x)$. In the proofs we follow some ideas developed earlier in [1] and [10].

In Section 4 we consider applications to some questions in measure metric spaces. We suggest a new approach to define local lower and upper dimensions. In terms of some bounds of these local dimensions we 
give a new characterization of the validity of some Zygmund-type integral inequalities involving measures $\mu B(x, r)$ of balls. Such inequalities arise in Muckehhoupt conditions for radial monotonic weights.

\section{Preliminaries}

2.1. Indices $m(w), m^{*}(w), m^{* *}(w)$ and $\quad M(w), M_{*}(w), M_{* *}(w)$ of functions $w \in \mathcal{W}$ depending on parameter. Let $X$ be a metric space with a positive measure $\mu$ (not necessarily satisfying the doubling condition) and $\Omega$ a bounded open set in $X$ with $\ell=\operatorname{diam} \Omega, 0<\ell<\infty$.

We will deal with functions $w(x, r)$ defined on $\Omega \times[0, \ell]$ which are almost increasing in variable $r$, and we will be interested in properties related to this almost monotonicity, uniform with respect to $x$.

We recall that a non-negative function $f$ on $[0, \ell]$ is said to be almost increasing (or almost decreasing ) if there exists a constant $C \geq 1$ such that $f\left(r_{1}\right) \leq C f\left(r_{2}\right)$ for all $0 \leq r_{1} \leq r_{2} \leq \ell$ (or $\ell \geq r_{1} \geq r_{2} \geq 0$, respectively).

Definition 2.1. By $\mathcal{W}=\mathcal{W}(\Omega \times[0, \ell])$ we denote the class of functions $w$ with the properties

1) $w \in L^{\infty}(\Omega \times[0, \ell])$;

2) $w(x, r)$ is continuous in $r \in[0, \ell]$ for any fixed $x \in \Omega$;

3) $w(x, 0)=0$, but

$$
\underset{x \in \Omega}{\operatorname{essinf}} w(x, r):=d_{0}(r)>0 \quad \text { for every } \quad r>0,
$$

where $\underset{x \in \Omega}{\operatorname{ess} \inf } w(x, r)$ is considered with respect to the measure $\mu$ on $X$;

4) for any fixed $x \in X$ the function $w(x, r)$ is almost increasing in $r$ with the uniform coefficient $1 \leq C_{w}<\infty$ not depending on $x$ :

$$
w\left(x, r_{1}\right) \leq C_{w} w\left(x, r_{2}\right), \quad 0 \leq r_{1} \leq r_{2} \leq \ell .
$$

In future for brevity we say that a function $w(x, r)$ is uniformly almost increasing in $r$ meaning that property (4) holds with $C_{w}$ not depending on $x$. When it is admitted that $w(x, r)$ is almost increasing at every point $x \in \Omega$ with $C_{w}$ which may depend on $x$, we say that $w(x, r)$ is pointwise almost increasing in $r$. Similar notions for almost decreasing functions are defined. 
Whenever necessary we always assume that $w(x, r)$ is defined as

$$
w(x, r)=w(x, \ell) \quad \text { for } \quad r \geq \ell .
$$

We also introduce the wider class

$$
\widetilde{\mathcal{W}}(X \times[0, \ell])=\left\{w(x, r): \exists a=a(w) \in \mathbb{R}^{1}\right.
$$

$$
\text { such that } \left.t^{a} w(x, t) \in \mathcal{W}(X \times[0, \ell])\right\} .
$$

Definition 2.2. Let $w \in \mathcal{W}$. The numbers

$$
\begin{aligned}
& m(w, x)=\sup _{r>1} \frac{\ln \left(\lim _{h \rightarrow 0} \frac{w(x, r h)}{w(x, h)}\right)}{\ln r}, \\
& M(w, x)=\inf _{r>1} \frac{\ln \left(\varlimsup_{h \rightarrow 0} \frac{w(x, r h)}{w(x, h)}\right)}{\ln r}
\end{aligned}
$$

will be referred to as the lower and upper Matuszewska-Orlicz indices of a function $w \in \mathcal{W}$ with respect to $r$.

We refer to [14], p. 20, [18], [10] for the Matuszewska-Orlicz indices $m(w), M(w)$ in the case where $w=w(r)$. The function

$$
W(x, r)=\varlimsup_{h \rightarrow 0} \frac{w(x, r h)}{w(x, h)}, \quad 0<r<\infty,
$$

involved in (2.5) and (2.6), is submultiplicative. Recall that a nonnegative everywhere finite function $f(t)$ defined on $(0, \infty)$, is called submultiplicative, if $f\left(t_{1} t_{2}\right) \leq f\left(t_{1}\right) f\left(t_{2}\right)$ for all $t_{1}, t_{1} \in(0, \infty)$. Following [13], Ch.II, formula (1.16) we call the function $W(x, r)$ a dilation of $w(x, r)$ (note that in [13] such a dilation was defined with $\sup _{h>0}$ instead of $\left.\varlimsup_{h \rightarrow 0}\right)$.

The lower index may be also written in terms of the dilation of $w(x, r)$ :

$$
m(w, x)=\sup _{0<r<1} \frac{\ln W(x, r)}{\ln r}=\lim _{r \rightarrow 0} \frac{\ln W(x, r)}{\ln r},
$$

which may be obtained by means of properties of submultiplicative functions. The following fact is known (see [13], Theorem 1.3). 
Lemma 2.3. If a function $f(r), 0<r<\infty$, is submultiplicative, then there exist $\lim _{r \rightarrow 0} \frac{\ln f(r)}{\ln r}$ and $\lim _{r \rightarrow \infty} \frac{\ln f(r)}{\ln r}$ and

$$
\lim _{r \rightarrow 0} \frac{\ln f(r)}{\ln r}=\sup _{0<r<1} \frac{\ln f(r)}{\ln r}, \quad \lim _{r \rightarrow \infty} \frac{\ln f(r)}{\ln r}=\inf _{r>1} \frac{\ln f(r)}{\ln r} .
$$

The change of variables $r \rightarrow \frac{1}{r}$ in (2.5) and Lemma 2.3 provide the coincidence of (2.5) with (2.8).

From (2.8) there also follows that

$$
m(w, x)=\lim _{r \rightarrow \infty} \frac{\ln \left(\frac{\lim }{h \rightarrow 0} \frac{w(x, r h)}{w(x, h)}\right)}{\ln r}, \quad M(w, x)=\lim _{r \rightarrow \infty} \frac{\ln W(x, r)}{\ln r} .
$$

For $w \in \mathcal{W}$ we have $0 \leq m(w, x) \leq M(w, x) \leq \infty$.

For the study of the Zygmund-Bary-Stechkin classes of functions depending on the parameter $x$, with the Zygmund condition uniform in $x$, the following numbers may be introduced

$$
m(w)=\sup _{r>1} \frac{\ln \left(\frac{\lim }{h \rightarrow 0} \operatorname{essinf} \frac{w(x, r h)}{w(x, h)}\right)}{\ln r}, \quad M(w)=\inf _{r>1} \frac{\ln \left(\varlimsup_{h \rightarrow 0}^{\operatorname{ess} \sup } \frac{w(x, r h)}{w(x, h)}\right)}{\ln r} .
$$

and the following their modifications which differ from (2.11) by the order of operations $\sup _{r>1}$ and $\underset{x \in \Omega}{\operatorname{essinf}}$ :

$$
m^{*}(w)=\sup _{r>1}\left(\operatorname{essinf}_{x \in \Omega} \frac{\ln \left(\frac{\lim }{h \rightarrow 0} \frac{w(x, r h)}{w(x, h)}\right)}{\ln r}\right),
$$

$$
M^{*}(w)=\inf _{r>1}\left(\operatorname{ess}_{x \in \Omega} \frac{\ln W(x, r)}{\ln r}\right)=\inf _{r>1}\left(\underset{x \in \Omega}{\operatorname{ess} \sup } \frac{\ln \left(\varlimsup_{h \rightarrow 0} \frac{w(x, r h)}{w(x, h)}\right)}{\ln r}\right),
$$

and

$$
m^{* *}(w):=\underset{x \in \Omega}{\operatorname{essinf}} m(w, x)=\underset{x \in \Omega}{\operatorname{essinf}}\left(\lim _{r \rightarrow 0} \frac{\ln W(x, r)}{\ln r}\right)
$$


and

$$
M^{* *}(w):=\underset{x \in \Omega}{\operatorname{ess} \sup } M(w, x)=\underset{x \in \Omega}{\operatorname{ess} \sup }\left(\lim _{r \rightarrow \infty} \frac{\ln W(x, r)}{\ln r}\right)
$$

Since always $\sup _{r} \operatorname{ess} \inf _{x} \leq \underset{x}{\operatorname{essinf}} \sup _{r}$, and $\liminf _{h \rightarrow 0} \underset{x \in \Omega}{\operatorname{essinf}} \leq \underset{x \in \Omega}{\operatorname{essinf}} \liminf _{h \rightarrow 0}$, we have

$$
m(w) \leq m^{*}(w) \leq m^{* *}(w) \quad \text { and } \quad M^{* *}(w) \leq M^{*}(w) \leq M(w) .
$$

We will mainly deal with the numbers $m(w), M(w)$ and in the sequel give sufficient conditions on $w$ for the coincidences $m(w)=m^{*}(w)=m^{* *}(w)$ and $M(w)=M^{*}(w)=M^{* *}(w)$.

Remark 2.4. The number $m(w)$ and $m^{*}(w)$ may be also represented as

$$
m(w)=\sup _{0<r<1}\left(\frac{\ln \left(\varlimsup_{h \rightarrow 0} \operatorname{esssup}_{x \in \Omega} \frac{w(x, r h)}{w(x, h)}\right)}{\ln r}\right)=\lim _{r \rightarrow 0}\left(\frac{\ln \left(\varlimsup_{h \rightarrow 0}^{\operatorname{ess} \sup } \frac{w(x, r h)}{w(x, h)}\right)}{\ln r}\right),
$$

$$
m^{*}(w)=\lim _{r \rightarrow 0}\left(\frac{\ln \left(\operatorname{esssup}_{x \in \Omega} \varlimsup_{h \rightarrow 0} \frac{w(x, r h)}{w(x, h)}\right)}{\ln r}\right)=\lim _{r \rightarrow 0}\left(\underset{x \in \Omega}{\operatorname{essinf}} \frac{\ln \left(\varlimsup_{h \rightarrow 0} \frac{w(x, r h)}{w(x, h)}\right)}{\ln r}\right) .
$$

Proof. Indeed, we transform $m^{*}(w)$ as follows:

$$
\begin{aligned}
m^{*}(w)=\sup _{r>1}\left(\frac{\ln \left(\operatorname{essinf}_{x \in \Omega} \frac{\lim }{h \rightarrow 0} \frac{w(x, h)}{w(x, h / r)}\right)}{\ln r}\right) & =\sup _{0<r<1}\left(\frac{\ln \left(\underset{x \in \Omega}{\operatorname{essinf}} \frac{\lim }{h \rightarrow 0} \frac{w(x, h)}{w(x, r h)}\right)}{\ln 1 / r}\right) \\
& =\sup _{0<r<1}\left(\frac{\ln \left(\operatorname{ess~sup~}_{x \in \Omega} \lim _{h \rightarrow 0} \frac{w(x, r h)}{w(x, h)}\right)}{\ln r}\right) .
\end{aligned}
$$

The function ess $\sup _{x \in \Omega} \varlimsup_{h \rightarrow 0} \frac{w(x, r h)}{w(x, h)}$ is obviously submultiplicative. Hence by Lemma 2.3 we arrive at (2.18). The arguments for $m(w)$ are similar. 
2.2. On the coincidence $m(w)=m^{*}(w)=m^{* *}(w)$. In Theorem 3.1 characterizing the generalized Bary-Stechkin class, we use only the numbers $m(w)$ and $m^{*}(w)$. However, in applications of these numbers to measuring lower and upper dimensions of metric measure spaces (see Section 4) the usage of the number $m^{* *}(w)$ seems to be more natural as the lower bound for local lower dimensions. So there arises an interest for sufficient conditions on $w(x, r)$ for the coincidence $m(w)=m^{*}(w)=m^{* *}(w)$.

We introduce the following definition.

Definition 2.5. A function $w \in \mathcal{W}(\Omega \times[0, \ell])$ will be said to satisfy condition $(\mathbb{A})$, if there exists an $\varepsilon>0$ such that

$$
\varlimsup_{h \rightarrow 0} \operatorname{esssup}_{x \in \Omega} \frac{w(x, r h)}{w(x, h)}=\underset{x \in \Omega}{\operatorname{ess} \sup _{h \rightarrow 0}} \varlimsup_{h \rightarrow m} \frac{w(x, r h)}{w(x, h)}
$$

for all $0<r<\varepsilon$ and its dilation (2.7) satisfies a similar assumption

$$
\underset{x \in \Omega}{\operatorname{essinf}} \lim _{r \rightarrow 0} \frac{\ln W(x, r)}{\ln r}=\lim _{r \rightarrow 0} \underset{x \in \Omega}{\operatorname{essinf}} \frac{\ln W(x, r)}{\ln r}
$$

(note that (2.19) and (2.20) always hold with $\geq$ instead of $=$ ).

Lemma 2.6. If a function $w(x, r)$ satisfies condition $(\mathbb{A})$, then $m(w)=$ $m^{*}(w)=m^{* *}(w)$.

Proof. In view of (2.19), from (2.17) and (2.18) it follows that $m(w) \geq m^{*}(w)$. Then $m(w)=m^{*}(w)$ by (2.16). Similarly, by $(2.20)$ we obtain that $m^{*}(w)=m^{* *}(w)$.

Of course, conditions (2.19)-(2.20) of Definition 2.5 are nothing else but just a reformulation of the fact that $m(w)=m^{*}(w)=m^{* *}(w)$. So we are interested in some easy to check sufficient conditions for $w$ to satisfy condition $(\mathbb{A})$.

The conditions of the next two lemmas are aimed, in particular, to the case where $w(x, r)=\mu B(x, r)$ may be a measure of balls in metric measure spaces.

Lemma 2.7. Let $w(x, r)$ have a form

$$
w(x, r)=A(x) r^{\psi(x, r)},
$$

where $A \in L^{\infty}(\Omega)$, essinf $|A(x)|>0$ and the function $\psi \in L^{\infty}(\Omega \times[0, \ell])$, continuous for small $0<r<\delta$, satisfies the uniform decay condition

$$
|\psi(x, r)-\psi(x, 0)| \leq C r^{\varepsilon}
$$


for some small $\varepsilon>0$. Then $m(w, x)=M(w, x)=\psi(x, 0)$. If $\psi$ satisfies also the condition

$$
\underset{x \in \Omega}{\operatorname{essinf}} \lim _{r \rightarrow 0} \psi(x, r)=\lim _{r \rightarrow 0} \underset{x \in \Omega}{\operatorname{essinf}} \psi(x, r),
$$

then $m(w)=m^{*}(w)=m^{* *}(w)=\underset{x \in \Omega}{\operatorname{essinf}} \psi(x, 0)$.

Proof. The proof is direct. Indeed,

$$
\frac{w(x, r h)}{w(x, h)}=r^{\psi(x, r h)} h^{\psi(x, r h)-\psi(x, h)} .
$$

From the uniform decay condition on $\psi(x, r)$ we easily obtain that

$$
\underset{x \in \Omega}{\operatorname{ess} \sup } \lim _{h \rightarrow 0} h^{\psi(x, r h)-\psi(x, h)}=\lim _{h \rightarrow 0} \underset{x \in \Omega}{\operatorname{ess} \sup } h^{\psi(x, r h)-\psi(x, h)}=1 .
$$

Hence the formulas $m(w, x)=M(w, x)=\psi(x, 0)$ follow.

To check the validity of (2.19), in view of (2.22) it is sufficient to verify that

$$
\lim _{h \rightarrow 0} \underset{x \in \Omega}{\operatorname{ess} \sup } r^{\psi(x, r h)} \leq \underset{x \in \Omega}{\operatorname{ess} \sup } \lim _{h \rightarrow 0} r^{\psi(x, r h)}
$$

for small $r$, which holds by (2.21). The validity of (2.20) is obvious since the dilation $W(x, r)$ of the function $w(x, r)=A(x) r^{\psi(x, r)}$ has the form $W(x, r)=r^{\psi(x, 0)}$

The following lemma provides sufficient conditions when $m(w, x)$ may be different from $M(w, x)$, which is important in those applications where lower and upper lower dimensions in measure metric spaces may be different.

Lemma 2.8. Let $w(x, r)$ have a form

$$
w(x, r)=A(x)[\varphi(r)]^{a(x)},
$$

where $A \in L^{\infty}(\Omega)$, essinf $|A(x)|>0, \varphi \in \mathcal{W}([0, \ell])$ and $a \in L^{\infty}(\Omega)$, essinf $a(x) \geq 0$. Then

$$
m(w, x)=a(x) m(\varphi), \quad M(w, x)=a(x) M(\varphi)
$$

and $m(w)=m^{*}(w)=m^{* *}(w)$.

Proof. We have $\frac{w(x, r h)}{w(x, h)}=\left(\frac{\varphi(r h)}{\varphi(h)}\right)^{a(x)}$. Then

$$
W(x, r)=[\Phi(r)]^{a(x)},
$$


where $\Phi(r)=\varlimsup_{h \rightarrow 0} \frac{\varphi(r h)}{\varphi(h)}$ is the dilation of the function $\varphi$. Hence the formulas (2.23) follow. By direct verification it is also easy to check that $w(x, r)$ satisfies condition $(\mathbb{A})$. Then $m(w)=m^{*}(w)=m^{* *}(w)$ by Lemma 2.6.

We refer to [20], where there are given various non-trivial examples of functions $\varphi(r)$ with non-coinciding local indices $m(\varphi), M(\varphi)$.

\subsection{On a generalized Zygmund-Bary-Stechkin class $\Phi_{\gamma}^{\beta}$.}

Definition 2.9. ([1], [5]) We say that a function $w(x, r) \in \mathcal{W}$ belongs to Zygmund-Bary-Stechkin class $\Phi$ in $r$ uniformly with respect to $x \in \Omega$, if

$$
\int_{0}^{r} \frac{w(x, t)}{t} d t \leq c w(x, r) \quad \text { and } \quad \int_{r}^{\ell} \frac{w(x, t)}{t^{2}} d t \leq c \frac{w(x, r)}{r}
$$

where $c=c(w)>0$ does not depend on $r \in(0, \ell]$ and $x \in \Omega$.

The class $\Phi$ of functions $w=w(r)$ was introduced in [1], where conditions (2.24) were imposed on monotonous functions in $\mathcal{W}$; we deal with almost monotonous functions. In the case where functions $w$ do not depend on $x$, the following statement characterizing the class $\Phi$ in terms of the indices $m(w)$ and $M(w)$, was proved in [18], p. 125.

Theorem 2.10. A function $w(r) \in \mathcal{W}([0, \ell])$ is in the Zygmund-BaryStechkin class $\Phi$ if and only if

$$
0<m(w) \leq M(w)<1,
$$

and for $w \in \Phi$ and any $\varepsilon>0$ there exist constants $c_{1}=c_{1}(w, \varepsilon)>0$ and $c_{2}=c_{2}(w, \varepsilon)>0$ such that

$$
c_{1} r^{M(w)+\varepsilon} \leq w(r) \leq c_{2} r^{m(w)-\varepsilon}, \quad 0 \leq r \leq \ell .
$$

Besides this, condition $m(w)>0$ is equivalent to the first inequality in (2.24), while condition $M(w)<1$ is equivalent to the second one.

We will obtain a similar statement for the general case treated in this paper and for the generalized Zygmund-Bary-Stechkin class $\Phi_{\gamma}^{\beta}$.

Let $\beta \geq 0, \gamma>0$. The following classes $\Phi_{\gamma}^{\beta}$, considered in [27] and [26], p. 253 , generalize the class $\Phi_{\gamma}^{0}$, introduced in [1].

Definition 2.11. By $\mathcal{Z}^{\beta}=\mathcal{Z}^{\beta}(\Omega \times[0, \ell])$, we denote the class of functions $w(x, r) \in \mathcal{W}$ satisfying the condition 


$$
\int_{0}^{r} \frac{w(x, t)}{t^{1+\beta}} d t \leq A \frac{w(x, r)}{r^{\beta}}, \quad 0<r \leq \ell
$$

and by $\mathcal{Z}_{\gamma}$ the class of functions $w(x, r) \in \mathcal{W}$ satisfying the condition

$$
\int_{r}^{\ell} \frac{w(x, t)}{t^{1+\gamma}} d t \leq A \frac{w(x, r)}{r^{\gamma}}, \quad 0<r \leq \ell
$$

where $A=A(w)>0$ does not depend on $r \in(0, \ell]$ and $x \in \Omega$. We denote $\Phi_{\gamma}^{\beta}=\mathcal{Z}^{\beta} \cap \mathcal{Z}_{\gamma}$.

In the sequel we refer to the above conditions as $\left(\mathbb{Z}^{\beta}\right)$ - and $\left(\mathbb{Z}_{\gamma}\right)$ conditions.

The class $\Phi_{\gamma}^{\beta}$ is nonempty if and only if $\beta<\gamma$, see Corollary 3.4 below.

Similarly to (2.25), we shall show that the condition $\beta<m(w) \leq M(w)<$ $\gamma$, with numbers $m(w)$ and $M(w)$ introduced in (2.11) is a characterization of the "uniform" class $\Phi_{\gamma}^{\beta}(\Omega \times[0, \ell])$, see Theorem 3.5.

2.4. Generalized Bary, Lozinskii and Stechkin conditions. Let $\beta, \gamma \in \mathbb{R}^{1}$. For functions $w \in \mathcal{W}(\Omega \times[0, \ell])$ we consider the following well known conditions (see [1], where such conditions were treated for $\beta=0$ and in the case of increasing functions $w=w(r)$ belonging to $\mathcal{W}([0, \ell]))$ :

1) Bary type conditions:

$$
\begin{aligned}
& \sum_{k=n+1}^{\infty} k^{\beta-1} w\left(x, \frac{1}{k}\right) \leq A n^{\beta} w\left(x, \frac{1}{n}\right), \\
& \sum_{k=1}^{n} k^{\gamma-1} w\left(x, \frac{1}{k}\right) \leq A n^{\gamma} w\left(x, \frac{1}{n}\right),
\end{aligned}
$$

where $A=A(w)>0$ does not depend on $n \in \mathbb{N}_{+}$and $x \in \Omega$,

2) Lozinskii type conditions:

there exists $C>1$ not depending on $x$ such that

$$
\underset{x \in \Omega}{\operatorname{essinf}} \frac{\lim }{r \rightarrow 0} \frac{w(x, C r)}{C^{\beta} w(x, r)}>1,
$$

there exists $C>1$ not depending on $x$ such that

$$
\underset{x \in \Omega}{\operatorname{ess} \sup } \varlimsup_{r \rightarrow 0} \frac{w(x, C r)}{C^{\gamma} w(x, r)}<1
$$


2a) strong Lozinskii type conditions:

there exists $C>1$ and $\varepsilon>0$ not depending on $x$ such that

$$
\underset{x \in \Omega}{\operatorname{essinf}} \inf _{0<r<\varepsilon} \frac{w(x, C r)}{C^{\beta} w(x, r)}>1,
$$

there exists $C>1$ and $\varepsilon>0$ not depending on $x$ such that

$$
\underset{x \in \Omega}{\operatorname{ess} \sup _{0}} \sup _{0<r<\varepsilon} \frac{w(x, C r)}{C^{\gamma} w(x, r)}<1,
$$

3) Stechkin type conditions:

there exists a $\delta>0$ such that $\frac{w(x, r)}{r^{\beta+\delta}}$ is uniformly a.i., $\quad\left(\mathbb{S}^{\beta}\right)$,

there exists a $\delta>0$ such that $\frac{w(x, r)}{r^{\gamma-\delta}}$ is uniformly a.d., $\quad\left(\mathbb{S}_{\gamma}\right)$,

4) $\mathbb{P}$ - conditions :

for any $\theta \in(0,1)$ there exists an integer $p=p(\theta)$ not depending on $x \in \Omega$ such that

$$
p^{\beta} w\left(x, \frac{\ell}{p n}\right)<\theta w\left(x, \frac{\ell}{n}\right),
$$

for any $\theta \in(0,1)$ there exists an integer $p=p(\theta)$ not depending on $x \in \Omega$ such that

$$
\theta p^{\gamma} w\left(x, \frac{\ell}{p n}\right)>w\left(x, \frac{\ell}{n}\right) .
$$

3. Characterization of functions $w \in \Phi_{\gamma}^{\beta}(\Omega \times[0, \ell])$ in terms of the indices $m(w)$ and $M(w)$

Theorem 3.1. Let $w \in \widetilde{\mathcal{W}}(\Omega \times[0, \ell])$. Then

I) condition $\left(\mathbb{L}^{\beta}\right)$ is equivalent to the inequality $m^{*}(w)>\beta$ and condition $\left(s \mathbb{L}^{\beta}\right)$ to the inequality $m(w)>\beta$;

II) conditions $\left(\mathbb{B}^{\beta}\right),\left(s \mathbb{L}^{\beta}\right),\left(\mathbb{Z}^{\beta}\right),\left(\mathbb{S}^{\beta}\right),\left(\mathbb{P}^{\beta}\right)$ are equivalent to each other: from the validity of one of them there follows the validity of all the others;

III) the statement in condition $\left(\mathbb{S}^{\beta}\right)$ holds with every $\delta<m(w)-\beta$. 
Proof. We may assume that $\beta \geq 0$, the case of negative $\beta$ being reduced to the case of positive $\beta$ by passing to the function $w_{1}(x, t)=t^{a} w(x, t)$ with $a>-\beta$.

I) According to (2.12) we have

$$
\begin{gathered}
m^{*}(w)>\beta \Longleftrightarrow \exists C>1: \frac{\ln \left(\underset{x \in \Omega}{\operatorname{essinf}} \liminf _{h \rightarrow 0} \frac{w(x, C h)}{w(x, h)}\right)}{\ln C}>\beta \\
\Longleftrightarrow \underset{x \in \Omega}{\operatorname{essinf}} \liminf _{h \rightarrow 0} \frac{w(x, C h)}{w(x, h)}>C^{\beta} \Longleftrightarrow\left(\mathbb{L}^{\beta}\right) .
\end{gathered}
$$

Similarly, by (2.11) we obtain

$$
\begin{aligned}
& m(w)>\beta \Longleftrightarrow \exists C>1: \frac{\ln \left(\liminf _{h \rightarrow 0} \underset{x \in \Omega}{\operatorname{essinf}} \frac{w(x, C h)}{w(x, h)}\right)}{\ln C}>\beta \\
& \Longleftrightarrow \liminf _{h \rightarrow 0} \underset{x \in \Omega}{\operatorname{essinf}} \frac{w(x, C h)}{w(x, h)}>C^{\beta} \Longleftrightarrow \exists \varepsilon>0 \text { such that } \\
& \underset{x \in \Omega}{\operatorname{essinf}} \frac{w(x, C h)}{w(x, h)}>C^{\beta} \text { for } 0<h<\varepsilon \Longleftrightarrow\left(s \mathbb{L}^{\beta}\right) .
\end{aligned}
$$

For part II) we prove the following chain

$$
\left(\mathbb{B}^{\beta}\right) \Longrightarrow\left(\mathbb{Z}^{\beta}\right) \Longrightarrow\left(s \mathbb{L}^{\beta}\right) \Longrightarrow\left(\mathbb{S}^{\beta}\right) \Longrightarrow\left(\mathbb{P}^{\beta}\right) \Longrightarrow\left(\mathbb{B}^{\beta}\right) .
$$

We suppose that $\beta>0$, modifications for the case $\beta=0$ are easy: power functions should be replaced by the logarithmic function under the corresponding integration. We take $\ell=1$ without loosing generality.

The implication $\left(\mathbb{B}^{\beta}\right) \Longrightarrow\left(\mathbb{Z}^{\beta}\right)$. Let $n=\left(\frac{1}{r}\right), \frac{1}{n+1}<r \leq \frac{1}{n}$ where $r \in(0,1]$. The inequality is valid

$$
\int_{0}^{r} \frac{w(x, t)}{t^{1+\beta}} d t \leq C_{w}\left(c(\beta) \sum_{k=n+2}^{\infty} k^{\beta-1} w\left(x, \frac{1}{k}\right)+d(\beta) r^{1-\beta} w(x, r)\right),
$$

where $c(\beta)=1$ when $\beta \leq 1$ and $c(\beta)=\frac{2^{\beta}-1}{\beta}$ when $\beta \geq 1$, and $d(\beta)=2$ when $\beta \leq 1$ and $d(\beta)=2 \cdot 3^{\beta-1}$ when $\beta \geq 1$. Indeed,

$$
\int_{0}^{r} \frac{w(x, t)}{t^{1+\beta}} d t=\sum_{k=n+2}^{\infty} \int_{\frac{1}{k+1}}^{\frac{1}{k}} \frac{w(x, t)}{t^{1+\beta}} d t+\int_{\frac{1}{n+2}}^{r} \frac{w(x, t)}{t^{1+\beta}} d t
$$




$$
\leq C_{w} \sum_{k=n+2}^{\infty} w\left(x, \frac{1}{k}\right) \frac{(k+1)^{\beta}-k^{\beta}}{\beta}+C_{w} w(x, r) \frac{(n+2)^{\beta}-r^{-\beta}}{\beta} .
$$

Since $(k+1)^{\beta}-k^{\beta} \leq c k^{\beta-1}$, where $c=\beta$ when $\beta \leq 1$ and $c=2^{\beta}-1$ when $\beta \geq 1$, and $(n+2)^{\beta} \leq(1+2 r)^{\beta} r^{-\beta}$, we obtain (3.1).

From (3.1) we get

$$
\frac{r^{\beta}}{w(x, r)} \int_{0}^{r} \frac{w(x, t)}{t^{1+\beta}} d t \leq C_{w}\left(c(\beta) \frac{r^{\beta}}{w(r)} \sum_{k=n+2}^{\infty} k^{\beta-1} w\left(\frac{1}{k}\right)+d(\beta) r^{1-\beta}\right)
$$

$$
\leq C_{w}\left(c(\beta) \frac{2^{\beta} C_{w}}{(n+1)^{\beta} w\left(\frac{1}{n+1}\right)} \sum_{k=n+2}^{\infty} k^{\beta-1} w\left(\frac{1}{k}\right)+d(\beta) r\right)
$$

whence the validity of $\left(\mathbb{Z}^{\beta}\right)$ follows by the validity of $\left(\mathbb{B}^{\beta}\right)$.

The implication $\left(\mathbb{Z}^{\beta}\right) \Longrightarrow\left(s \mathbb{L}^{\beta}\right)$. Given $\left(\mathbb{Z}^{\beta}\right)$, that is,

$$
\int_{0}^{r} \frac{w(x, t) d t}{t^{1+\beta}} \leq A \frac{w(x, r)}{r^{\beta}}, \quad 0<r \leq 1,
$$

we shall show that condition $\left(s \mathbb{L}^{\beta}\right)$, that is, the condition

$$
\underset{x \in \Omega}{\operatorname{essinf}} \inf _{0<r<\varepsilon_{0}} \frac{w(x, C r)}{w(x, r)}>C^{\beta}
$$

holds with $C=2 e^{2 A M}(>2)$ and $\varepsilon_{0}=\frac{1}{2} e^{-2 A M}$, where $M=\frac{\beta 2^{\beta} A C_{w}}{2^{\beta}-1}$.

First we shall show that

$$
\frac{w(x, \xi)}{\xi^{\beta}} \leq M \frac{w(x, r)}{r^{\beta}},
$$

for all $\xi \in(0,1), r \in(0,1)$ such that $\frac{\xi}{r} \leq \frac{1}{2}$ ("ersatz" of the almost monotonicity). Indeed, from (3.3) it follows that $\int_{\xi}^{r} \frac{w(x, t) d t}{t^{1+\beta}} \leq A \frac{w(x, r)}{r^{\beta}}$ whence $\frac{w(x, \xi)}{C_{w}} \int_{\xi}^{r} \frac{d t}{t^{1+\beta}} \leq A \frac{w(x, r)}{r^{\beta}}$. Hence $\frac{w(x, \xi)}{\xi^{\beta}} \cdot \frac{1-\left(\frac{\xi}{r}\right)^{\beta}}{\beta} \leq A C_{w} \frac{w(x, r)}{r^{\beta}}$ which yields (3.5) since $\frac{\xi}{r} \leq \frac{1}{2}$.

Now the key moment is that we repeat the same idea once more. For all $\xi>2 \eta$ from (3.3) we obtain $\int_{2 \eta}^{\xi} \frac{w(x, t) d t}{t^{1+\beta}} \leq A \frac{w(x, \xi)}{\xi^{\beta}}$. Then $\frac{1}{M} \frac{w(x, \eta)}{\eta^{\beta}} \int_{2 \eta}^{\xi} \frac{d t}{t} \leq$ 
$A \frac{w(x, \xi)}{\xi^{\beta}}$ by $(3.5)$ or

$$
\frac{w(x, \eta)}{\eta^{\beta}} \leq \frac{A M}{\ln \frac{\xi}{2 \eta}} \cdot \frac{w(x, \xi)}{\xi^{\beta}}
$$

We choose now a relation between $\eta$ and $\xi$ in the following way: $\frac{\xi}{\eta}=C=$ $2 e^{2 A M}$. Then $\frac{A M}{\ln \frac{\xi}{2 \eta}}=\frac{1}{2}$ and from (3.6) we obtain $\frac{w(x, \eta)}{\eta^{\beta}} \leq \frac{1}{2} \frac{w(x, C \eta)}{(C \eta)^{\beta}}$ for $\eta$ sufficiently small $\left(0<\eta<\frac{1}{2} e^{-2 A M}\right)$ and all $x$. Therefore, $w(x, C \eta) \geq 2 C^{\beta} w(x, \eta)$. Hence $\liminf _{\eta \rightarrow 0} \underset{x \in \Omega}{\operatorname{essinf}} \frac{w(x, C \eta)}{w(x, \eta)} \geq 2 C^{\beta}>C^{\beta}$, that is, $\left(s \mathbb{L}^{\beta}\right)$ has been obtained.

The implication $\left(s \mathbb{L}^{\beta}\right) \Longrightarrow\left(\mathbb{S}^{\beta}\right)$. Let $\left(s \mathbb{L}^{\beta}\right)$ be valid: there exists a $C>1$ such that

$$
\nu=\nu(C, \varepsilon):=\underset{x \in \Omega}{\operatorname{essinf}} \inf _{0<r<\varepsilon} \frac{w(x, C r)}{C^{\beta} w(x, r)}>1 .
$$

We shall show that the function $\frac{w(x, r)}{r^{\beta+\delta}}$ with every

$$
\delta<m(w)
$$

is uniformly a.i. in $r$.

Let $0<\varepsilon_{1}<\nu-1$. From (3.7) it follows that for every such $\varepsilon$ there exists an $r_{1}$ not depending on $x$ such that

$$
\frac{w(x, C r)}{C^{\beta} w(x, r)} \geq \nu-\varepsilon_{1}(>1) \quad \text { for } \quad 0<r \leq r_{1} .
$$

We choose $\delta=\delta\left(C, \varepsilon, \varepsilon_{1}\right)=\frac{\ln \left(\nu-\varepsilon_{1}\right)}{\ln C}>0$ and show that $\frac{w(x)}{x^{\beta+\delta}}$ is almost increasing under this choice of $\delta$. With this $\delta$, inequality (3.9) takes the form

$$
\frac{w(x, r)}{r^{\beta+\delta}} \leq \frac{w(x, C r)}{(C r)^{\beta+\delta}}, \quad 0<r \leq r_{1} .
$$

Now, for arbitrary $0<\rho<r \leq r_{1}$ we choose an integer $N$ by the condition $C^{N} \rho \leq r<C^{N+1} \rho\left(N=\left(\log _{C} \frac{r}{\rho}\right)\right)$. Then by (3.10) we get

$$
\frac{w(x, \rho)}{\rho^{\beta+\delta}} \leq \frac{w(x, C \rho)}{(C \rho)^{\beta+\delta}} \leq \cdots \leq \frac{w\left(x, C^{N} \rho\right)}{\left(C^{N} \rho\right)^{\beta+\delta}} .
$$


Since $w(x, r)$ is a.i., we obtain

(3.11)

$\frac{w(x, \rho)}{\rho^{\beta+\delta}} \leq C_{w} C^{\beta+\delta} \frac{w(x, r)}{\left(C^{N+1} \rho\right)^{\beta+\delta}} \leq C_{w} C^{\beta+\delta} \frac{w(x, r)}{r^{\beta+\delta}}=C_{w} C^{\beta}(\nu-\varepsilon) \frac{w(x, r)}{r^{\beta+\delta}}$.

Thus $\frac{w(x, r)}{r^{\beta+\delta}}$ is almost increasing in $r$ on $\left[0, r_{1}\right]$. Since any positive function bounded from below is a.i, from condition (2.1) it follows that $\frac{w(x, r)}{r^{\beta+\delta}}$ is almost increasing on $[0, \ell]$. This fact has been proved for any $\delta$ such that

$$
0<\delta=\frac{\ln \left(\nu-\varepsilon_{1}\right)}{\ln C}=\frac{\ln \nu}{\ln C}-\varepsilon_{2}
$$

with an arbitrarily small $\varepsilon_{2}$. This means that one may take any $\delta$ satisfying (3.12) with an arbitrary $C>1$ such that the inequality $\nu=\nu(C, \varepsilon)>1$ in (3.7) is valid, that is,

$$
\delta<\sup _{C>1} \frac{\ln \nu(C, \varepsilon)}{\ln C}=\sup _{C>1} \frac{\ln \left(\operatorname{essinf}_{x \in \Omega} \inf _{0<r<\varepsilon} \frac{w(x, C r)}{C^{\beta} w(x, r)}\right)}{\ln C} .
$$

Since $\varepsilon>0$ is arbitrarily small, the right hand side in (3.13) may be arbitrarily close to $m(w)$ in case $m(w)<\infty$. If $m(w)=\infty$, then $\delta$ may be taken arbitrarily large. In both cases one may take an arbitrary $\delta<m(w)$.

The implication $\left(\mathbb{S}^{\beta}\right) \Longrightarrow\left(\mathbb{P}^{\beta}\right)$. Let $\frac{w(x, r)}{r^{\beta+\delta}}$ be almost increasing for some $\delta>0$ : $\frac{w(x, r)}{r^{\beta+\delta}} \leq B \frac{w(x, \rho)}{\rho^{\beta+\delta}}$ for $0<r \leq \rho \leq 1$. We here choose $r=\frac{1}{p n}$ and $\rho=\frac{1}{n}$ and obtain $p^{\beta+\delta} w\left(\frac{1}{p n}\right) \leq B w\left(\frac{1}{n}\right), n=1,2,3, \ldots$ where the integer $p$ is to be chosen. Given an arbitrary $\theta \in(0,1)$, we choose $p=p(\theta)>\left(\frac{B}{\theta}\right)^{\frac{1}{\delta}}$ so that $p^{\delta}>\frac{B}{\theta}$ and then $p^{\beta} w\left(\frac{1}{p n}\right)<\theta w\left(\frac{1}{n}\right)$.

The implication $\left(\mathbb{P}^{\beta}\right) \Longrightarrow\left(\mathbb{B}^{\beta}\right)$. We have

$$
\sum_{k=n+1}^{\infty} k^{\beta-1} w\left(x, \frac{1}{k}\right)=\sum_{s=0}^{\infty} \sum_{k=p^{s} n+1}^{p^{s+1} n} k^{\beta-1} w\left(x, \frac{1}{k}\right)
$$

for any choice of the integer $p$. Since the function $w(x, r)$ is almost increasing in $r$, we then get

$$
\sum_{k=n+1}^{\infty} k^{\beta-1} w\left(x, \frac{1}{k}\right) \leq C_{w} \sum_{s=0}^{\infty} w\left(x, \frac{1}{p^{s} n}\right) \sum_{k=p^{s} n+1}^{p^{s+1} n} k^{\beta-1} .
$$


Obviously, $\sum_{k=m}^{n} k^{\beta-1}=\sum_{k=m}^{n} k^{\beta-1} \int_{k-1}^{k} d x \leq c \sum_{k=m}^{n} \int_{k-1}^{k} x^{\beta-1} d x$ with some constant $c>0$ for all $k \geq 2(c=1$ when $\beta \leq 1)$. Therefore, $\sum_{k=m}^{n} k^{\beta-1} \leq c \int_{m-1}^{n} x^{\beta-1}=c \frac{n^{\beta}-(m-1)^{\beta}}{\beta}$ and then

$$
\sum_{k=n+1}^{\infty} k^{\beta-1} w\left(x, \frac{1}{k}\right) \leq c C_{w} \frac{n^{\beta}\left(p^{\beta}-1\right)}{\beta} \sum_{s=0}^{\infty} p^{\beta s} w\left(x, \frac{1}{p^{s} n}\right) .
$$

By condition $\left(\mathbb{P}^{\beta}\right)$, for any $\theta \in(0,1)$ we can choose an integer $p$ such that $w\left(x, \frac{1}{p^{s} n}\right) \leq\left(\frac{\theta}{p^{\beta}}\right)^{s} w\left(x, \frac{1}{n}\right)$. Consequently,

$$
\sum_{k=n+1}^{\infty} k^{\beta-1} w\left(x, \frac{1}{k}\right) \leq c_{1} n^{\beta} w\left(x, \frac{1}{n}\right) \sum_{s=0}^{\infty} \theta^{s},
$$

with $c_{1}=\frac{C_{w}}{\beta}\left(p^{\beta}-1\right)$, that is, $\left(\mathbb{B}^{\beta}\right)$ holds.

III). This part was already proved under the passage $\left(s \mathbb{L}^{\beta}\right) \Longrightarrow\left(\mathbb{S}^{\beta}\right)$, see (3.8).

Theorem 3.2. Let $w \in \widetilde{\mathcal{W}}(\Omega \times[0, \ell])$. Then

I) condition $\left(\mathbb{L}_{\gamma}\right)$ is equivalent to the inequality $M_{*}(w)<\gamma$ and condition $\left(s \mathbb{L}_{\gamma}\right)$ to the inequality $M(w)<\gamma$;

II) conditions $\left(\mathbb{B}_{\gamma}\right),\left(s \mathbb{L}_{\gamma}\right),\left(\mathbb{Z}_{\gamma}\right),\left(\mathbb{S}_{\gamma}\right),\left(\mathbb{P}_{\gamma}\right)$ are equivalent to each other: from the validity of one of them there follows the validity of all the others;

III) the statement in condition $\left(\mathbb{S}^{\gamma}\right)$ holds with every $\delta<\gamma-M(w)$.

The proof of Theorem 3.2 is symmetrical to that of Theorem 3.1 and thereby is omitted.

Remark 3.3. Statement II) of Theorems 3.1 and 3.2 was proved in [1] in the case when $\beta=0$ and functions $w=w(r)$ were monotonous. A modification of the proof from [1] adjusted for the case of $\beta \geq 0$ and almost increasing functions $w(r)$ was given in [10]. For the case of functions $w(x, r)$ depending on a parameter $x$ belonging to a metric measure space, we followed mainly the arguments of the proof in [10], with modifications everywhere, where the uniformness of various estimates with respect to $x$ was needed. Theorems 3.1 and 3.2 for almost increasing functions $w=w(r)$ were earlier proved for $\beta=0$ and $\gamma=1$ in [18]. 
Corollary 3.4. The class $\Phi_{\gamma}^{\beta}=\Phi_{\gamma}^{\beta}(\Omega \times[0, \ell]), \beta \geq 0, \gamma>0$ is non-empty if and only if $\beta<\gamma$.

Proof. Indeed, from statements I)-II) of Theorems 3.1 and 3.2 it follows that if $w \in \Phi_{\gamma}^{\beta}$, then $m(w)>\beta$ and $M(w)<\gamma$. But $m(w) \leq M(w)$, so that $\beta<\gamma$. Inversely, if $\beta<\gamma$, then the power function $w=r^{a}$ with $\beta<a<\gamma$ obviously belongs to $\Phi_{\gamma}^{\beta}$.

Theorem 3.5. Let $w \in \mathcal{W}$ and $0 \leq \beta<\gamma<\infty$. Then

$$
w \in \Phi_{\gamma}^{\beta}(\Omega \times[0, \ell]) \Longleftrightarrow \beta<m(w) \leq M(w)<\gamma
$$

and for $w \in \Phi_{\gamma}^{\beta}$ and any $\varepsilon>0$ there exist constants $c_{1}=c_{1}(\varepsilon)>0$ and $c_{2}=c_{2}(\varepsilon)>0$ not depending on $x \in \Omega$ such that

$$
c_{1} r^{M(w)+\varepsilon} \leq w(x, r) \leq c_{2} r^{m(w)-\varepsilon}, \quad 0 \leq r \leq \ell .
$$

Proof. Indeed, the equivalence in (3.14) follows from statement III) and I) of Theorems 3.1 and 3.2 .

To get at inequalities (3.15), it suffices to observe that the functions

$$
\frac{w(x, r)}{r^{m(w)-\varepsilon}} \text { and } \frac{w(x, r)}{r^{M(w)+\varepsilon}}
$$

are uniformly almost increasing and decreasing, respectively, for any $\varepsilon>0$ according to statement III) of Theorems 3.1 and 3.2, and any uniformly almost increasing or almost decreasing function is uniformly bounded from above or from below, respectively.

The following theorem characterizes the conditions $\left(\mathbb{S}^{\beta}\right)$ and $\left(\mathbb{S}_{\gamma}\right)$ in terms of the indices $m(w)$ and $M(w)$.

Theorem 3.6. For any function $w \in \mathcal{Z}^{\beta}$ its lower index bound $m(w)$ may be calculated by the formula

$$
m(w)=\sup \left\{\delta>\beta: \frac{w(x, r)}{r^{\delta}} \text { is uniformly a.i. }\right\},
$$

while for any $w \in \mathcal{Z}_{\gamma}$ its upper index bound $M(w)$ is calculated by the formula

$$
M(w)=\inf \left\{\delta \in(0, \gamma): \frac{w(x, r)}{r^{\delta}} \text { is uniformly a.d. }\right\}
$$


Proof. Let $a=\sup \left\{\delta>\beta: \frac{w(x, r)}{r^{\delta}}\right.$ is a.i. $\}$. By statement III) of Theorem 3.1, $m(w) \leq a$. We have to prove that $m(w)=a$. Suppose to the contrary that $m(w)<a$. Then the function $w_{1}(x, r)=\frac{w(x, r)}{r m(w)}$ is also almost increasing and $w_{1}(x, 0)=0$ since the function $\frac{w(x, r)}{r^{m(w)+\delta}}$ is also uniformly almost increasing with $0<\delta<a-m(w)$. Therefore, $w_{1} \in \mathcal{W}(\Omega \times[0, \ell])$. The function $w_{1}(x, r)$ satisfies condition $\mathbb{S}^{0}=\left.\mathbb{S}^{\beta}\right|_{\beta=0}$. Then, by statement I) of Theorem 3.1, $m\left(w_{1}\right)>0$, which is impossible since $m\left(w_{1}\right)=m(w)-m(w)=0$.

Similarly, formula (3.17) is obtained.

\section{Applications}

The results presented in Section 3 may be used in the study of the mapping properties of singular and potential operators in the generalized Hölder spaces $H^{\omega(x, \cdot)}(X)$, with variable characteristic, where $X$ in general may be a metric measure space, defined by the norm

$$
\|f\|_{H^{\omega(x, \cdot)}(X)}=\|f\|_{C(X)}+\sup _{x \in X} \sup _{0<h<\ell} \frac{\omega(f, x, h)}{\omega(x, h)}, \quad \ell=\operatorname{diam} X,
$$

where by

$$
\omega(f, x, h)=\sup _{\substack{y \in X: \\|x-y|<h}}|f(x)-f(y)|
$$

we denote the local continuity modulus of a function $f$ at the point $x$, and $\omega(x, h)$ stands for the dominant of these moduli, defining the Hölder space (without fear of confusion we use the same letter $\omega$ for this dominant). Note that such generalized Hölder spaces with variable dominant of the modulus of continuity were studied in the case where $X=\mathbb{S}^{n-1}$ in [30], [31].

We do not consider such applications in this paper, but dwell on applications of the indices depending on parameter to the problem of measuring local dimensions in metric measure spaces and to characterization of some integral inequalities involving measures of balls in metric measure spaces. Note that in what follows metric measure spaces are not assumed to satisfy the doubling condition.

4.1. Local variable dimensions. Let $(X, d, \mu)$ be a metric measure space with quasidistance $d: X \times X \rightarrow \mathbb{R}^{1}$ satisfying the standard assumptions:

$$
d(x, y) \geq 0, \quad d(x, y)=0 \Longleftrightarrow x=y, \quad d(x, y)=d(y, x),
$$




$$
d(x, y) \leq a[d(x, z)+d(z, y)], \quad a \geq 1,
$$

and a non-negative measure $\mu$, and let $B(x, r)=\{y \in X: d(x, y)<r\}$. We refer to [8], [4] for basics on metric measure spaces. In the particular case where for every point $x \in X$ there exists a positive number $s=s(x)$ such that

$$
C_{1} r^{s(x)+\varepsilon} \leq \mu B(x, r) \leq C_{2} r^{s(x)-\varepsilon},
$$

for every positive $\varepsilon>0$, where the constants $C_{1}>0, C_{2}>0$ in general depend on $x$ and $\varepsilon$, then the space $X$ may be said to have a local dimension at a point $x$ calculated by the formula

$$
\operatorname{dim}_{X}(x)=\lim _{r \rightarrow 0} \frac{\ln \mu B(x, r)}{\ln r} .
$$

In the general case, the lower and upper local dimensions

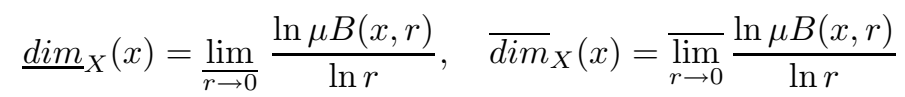

may be attributed to a point $x$. We refer to [3] (p. 25), [6], [7], where such local dimensions were introduced and/or used. Formulas (4.4) reflect the case where instead of (4.3) one has

$$
C_{1} r^{\overline{\operatorname{dim}}_{X}(x)+\varepsilon} \leq \mu B(x, r) \leq C_{2} r \frac{\operatorname{dim}}{X}(x)-\varepsilon \quad \text { as } \quad r \rightarrow 0,
$$

for arbitrary $\varepsilon>0$ (with $C_{1}$ and $C_{2}$ in general depending not only on $x$, but on $\varepsilon$ as well).

Comparison with property (3.15) of almost monotonic functions allows us to suggest new formulas for local lower and upper dimensions of metric measure spaces, based the technique of the lower and upper indices adjusted for nonnegative monotonic (or almost monotonic) functions and presented in Section 2-3. Namely, the lower and upper local dimensions at a point $x$ may be introduced as lower and upper indices $m(\mu B(x, r)), M(\mu B(x, r))$ of the measure of the ball $B(x, r)$. In the case when $X$ is bounded we introduce the local lower and upper dimensions in the form

$$
\underline{\mathfrak{d i m}}_{X}(x):=m\left(\mu B_{x}\right)=\sup _{r>1} \frac{\ln \left(\frac{\lim }{t \rightarrow 0} \frac{\mu B(x, r t)}{\mu B(x, t)}\right)}{\ln r},
$$




$$
\overline{\mathfrak{d i m}}_{X}(x):=M\left(\mu B_{x}\right)=\inf _{r>1} \frac{\ln \left(\varlimsup_{t \rightarrow 0} \frac{\mu B(x, r t)}{\mu B(x, t)}\right)}{\ln r},
$$

According to (2.8) and Lemma 2.3, we can also write the dimensions $\mathfrak{d i m}_{X}(x)$ and $\overline{\mathfrak{d i m}}_{X}(x)$ in the form

$$
\underline{\mathfrak{d i m}}_{X}(x)=\sup _{0<r<1} \frac{\ln \mathcal{M}(x, r)}{\ln r}=\lim _{r \rightarrow 0} \frac{\ln \mathcal{M}(x, r)}{\ln r}
$$

and

$$
\overline{\mathfrak{d i m}}_{X}(x)=\inf _{r>1} \frac{\ln \mathcal{M}(x, r)}{\ln r}=\lim _{r \rightarrow \infty} \frac{\ln \mathcal{M}(x, r)}{\ln r},
$$

where $\mathcal{M}(x, r)=\varlimsup_{t \rightarrow 0} \frac{\mu B(x, r t)}{\mu B(x, t)}$ is a submultiplicative function: $\mathcal{M}\left(x, r_{1} r_{2}\right) \leq$ $\mathcal{M}\left(x, r_{1}\right) \mathcal{M}\left(x, r_{2}\right)$.

An advantage of the usage of these local dimensions in comparison with dimensions (4.4) is in the fact that just in terms of the dimensions $\underline{\mathfrak{d i m}}_{X}(x)$ and $\overline{\mathfrak{d i m}}_{X}(x)$ it is possible to characterize some integral inequalities involving the measures $\mu B(x, r)$, which appear when one considers the Muckenhoupt type $A_{p}$-condition on metric measure spaces, see Theorem 4.2 .

The following statement is obvious.

Lemma 4.1. The condition

$$
\varlimsup_{t \rightarrow 0} \frac{\mu B(x, r t)}{\mu B(x, t)} \leq \mu B(x, r)
$$

at a point $x \in X$ for $0<r<\varepsilon$ with some $\varepsilon>0$ implies $\underline{\operatorname{dim}}_{X}(x) \leq$ $\underline{\mathfrak{d i m}}_{X}(x)$ and the inverse inequality implies $\underline{\operatorname{dim}}_{X}(x) \geq \underline{\mathfrak{d i m}}_{X}(x)$ and a similar statement with $\varlimsup_{t \rightarrow 0}$ replaced by $\lim _{t \rightarrow 0}$ holds for $\overline{\operatorname{dim}}_{X}(x)$ and $\overline{\mathfrak{d i m}}_{X}(x)$.

4.2. On lower and upper dimensions of a measure metric space. Basing on the numbers introduced in (2.11),(2.12) and (2.14), we may define the following versions for the notion of the lower dimension of the space $(X, d, \mu)$ :

$$
\underline{\mathfrak{d i m}}(X)=\sup _{r>1} \frac{\ln \left(\lim _{h \rightarrow 0} \operatorname{essinf} \frac{\mu B(x, r h)}{\mu B(x, h)}\right)}{\ln r}=\lim _{r \rightarrow 0}\left(\frac{\ln \left(\varlimsup_{h \rightarrow 0}^{\operatorname{esssup}} \underset{x \in \Omega}{\left.\operatorname{en} \frac{\mu B(x, r h)}{\mu B(x, h)}\right)}\right)}{\ln r}\right),
$$


$(4.11)$

$\underline{\mathfrak{d i m}}^{*}(X)=\sup _{r>1}\left(\underset{x \in \Omega}{\operatorname{essinf}} \frac{\ln \left(\frac{\lim }{h \rightarrow 0} \frac{\mu B(x, r h)}{\mu B(x, h)}\right)}{\ln r}\right)=\lim _{r \rightarrow 0}\left(\underset{x \in \Omega}{\operatorname{essinf}} \frac{\ln \left(\varlimsup_{h \rightarrow 0} \frac{\mu B(x, r h)}{\mu B(x, h)}\right)}{\ln r}\right)$,

$$
\underline{\mathfrak{d i m}}^{* *}(X)=\underset{x \in X}{\operatorname{ess} \inf } \underline{\mathfrak{d i m}}_{X}(x)=\underset{x \in \Omega}{\operatorname{ess} \inf _{\text {fup }}} \sup _{r>1} \frac{\ln \left(\frac{\lim }{h \rightarrow 0} \frac{\mu B(x, r h)}{\mu B(x, h)}\right)}{\ln r} .
$$

Similarly one may write down a similar version for the upper dimensions:

$$
\overline{\mathfrak{d i m}}(X):=\inf _{r>1} \frac{\ln \left(\varlimsup_{t \rightarrow 0} \operatorname{essinf}_{x \in \Omega} \frac{\mu B(x, r t)}{\mu B(x, t)}\right)}{\ln r}
$$

and analogously for $\overline{\mathfrak{d i m}} *(X)$ and $\overline{\mathfrak{d i m}} * *(X)=\underset{x \in X}{\operatorname{essinf}} \overline{\mathfrak{d i m}}_{X}(x)$, basing on definitions in (2.11), (2.13) and (2.15).

4.3. On some integral inequalities involving the measure $\mu B(x, r)$. In the most of the statements in the sequel we assume that the measure $\mu$ satisfies the following assumptions:

i) $\mu$ is non-atomic;

ii) the measures $\mu B(x, r)$ of balls are continuous in $r$ for every $x \in X$,

iii) the condition

$$
\underset{x \in X}{\operatorname{essinf}} \mu B(x, r)>0 \quad \text { for every } \quad r>0
$$

is fulfilled.

As is known, condition (4.14) is always fulfilled when $X$ is bounded and the measure $\mu$ is doubling, which follows from the property

$$
\frac{\mu B(x, R)}{\mu B(x, r)} \leq C\left(\frac{R}{r}\right)^{\log _{2} C_{\mu}}, \quad 0<r \leq R<\infty
$$

of doubling measures, where $C_{\mu}$ is the constant from the doubling condition.

From items I)-II) of Theorems 3.1 and 3.2 we derive the following statement.

Theorem 4.2. Let $0<\ell<\infty, \beta \geq 0, \gamma>0$ and the measure $\mu$ satisfy assumptions i)-iii). The inequalities 


$$
\int_{0}^{h} \frac{\mu B(x, r)}{r^{1+\beta}} d r \leq C \frac{\mu B(x, h)}{h^{\beta}}, \quad \int_{h}^{\ell} \frac{\mu B(x, r)}{r^{1+\gamma}} d t \leq C \frac{\mu B(x, h)}{h^{\gamma}}, \quad 0<h \leq \ell,
$$

with constants $C_{1}>0, C_{2}>0$ not depending on $h$ and $x$ hold if and only if

$$
\underline{\mathfrak{d i m}}(X)>\beta \quad \text { and } \quad \overline{\mathfrak{d i m}}(X)<\gamma,
$$

respectively.

In applications to weighted estimations of maximal, singular and potential operators on metric measure spaces the following more general integral inequalities for radial weights $w\left[d\left(x, x_{0}\right)\right]$ and measures $\mu B(x, r)$

$$
\begin{gathered}
\int_{0}^{h} \frac{\mu B(x, r) u(r)}{r} d r \leq C u(h) \mu B(x, h), \\
\int_{0}^{h} \frac{\mu B(x, r)}{r v(r)} d r \leq C \frac{\mu B(x, h)}{v(h)}
\end{gathered}
$$

are of importance, where $u, v$ are almost increasing functions and $0<h \leq$ $\ell<\infty$. (For simplicity, we do not take functions $u$ and $v$ depending on the parameter $x$ ). The next theorem provides sufficient conditions for their validity in terms of the indices of the weight and the lower dimension of the space.

Theorem 4.3. Let $u, v \in \widetilde{\mathcal{W}}([0, \ell])$. Under assumptions $i)$-iii), the conditions

$$
m(u)>-\underline{\mathfrak{d i m}}(X) \quad \text { and } \quad M(v)<\underline{\mathfrak{d i m}}(X)
$$

are sufficient for inequalities (4.16)-(4.17) to hold, respectively.

Proof. It suffices to apply Theorem 3.1 and make use of the following properties of the index numbers

$$
m\left(w_{1} w_{2}\right) \geq m\left(w_{1}\right)+m\left(w_{2}\right), \quad m\left(\frac{1}{v}\right)=-M(v)
$$

of functions $w_{1}, w_{2}, v \in \widetilde{\mathcal{W}}$. 
4.4. The case of unbounded metric measure spaces. When $X$ is unbounded, conditions (4.18) with $\ell=\infty$ do not guarantee the validity of inequalities (4.16)-(4.17): an information about the behavior of functions $u, v$ and the measures $\mu B(x, r)$ as $r \rightarrow \infty$, is also needed. Characteristic of the measures $\mu B(x, r)$, required for this goal, may be introduced in terms similar to the above introduced dimensions:

$$
\underline{\operatorname{dim}}_{X}(\infty)=\sup _{0<r<1} \frac{\ln \mathcal{M}_{\infty}(x, r)}{\ln r}=\lim _{r \rightarrow 0} \frac{\ln \mathcal{M}_{\infty}(x, r)}{\ln r},
$$

and

$$
\overline{\operatorname{dim}}_{X}(\infty)=\inf _{r>1} \frac{\ln \mathcal{M}_{\infty}(x, r)}{\ln r}=\lim _{r \rightarrow \infty} \frac{\ln \mathcal{M}_{\infty}(x, r)}{\ln r}
$$

where

$$
\mathcal{M}_{\infty}(x, r)=\varlimsup_{t \rightarrow \infty} \frac{\mu B(x, r t)}{\mu B(x, t)} .
$$

The numbers $\underline{\operatorname{dim}}_{X}(\infty), \overline{\operatorname{dim}}_{X}(\infty)$ as introduced in (4.20)-(4.21) do not depend on $x \in X$, which is proved in the following lemma. Note that they are not limits of dimensions $\underline{\operatorname{dim}}_{X}(x)$ and $\overline{\operatorname{dim}}_{X}(x)$ or dimensions $\underline{\mathfrak{d i m}}_{X}(x), \overline{\mathfrak{d i m}}_{X}(x)$ as $x \rightarrow \infty$ : simple examples show that these limits may not exist, while $\underline{\operatorname{dim}}_{X}(\infty), \overline{\operatorname{dim}}_{X}(\infty)$ always exist as finite or infinite number, and when those limits exist they do not necessarily coincide with $\underline{\operatorname{dim}}_{X}(\infty), \overline{\operatorname{dim}}_{X}(\infty)$.

Lemma 4.4. Let $(X, d, \mu)$ be an unbounded metric measure space. Then the functions

$$
\lim _{r \rightarrow \infty} \frac{\ln \mathcal{M}_{\infty}(x, r)}{\ln r}, \lim _{r \rightarrow 0} \frac{\ln \mathcal{M}_{\infty}(x, r)}{\ln r}
$$

do not depend on $x$. In the case where the measure $\mu$ satisfies also assumption ii) and $d(x, y)$ is a distance, that is, $a=1$ in (4.2), then even the function $\mathcal{M}_{\infty}(x, r)$ does not depend on $x$.

Proof. Let $x, y$ be arbitrary points of $X$ and let $t>a d(x, y)$. By the triangle inequality (4.2) we have

$$
B(x, r) \subset B(y, a r+a d(x, y))
$$

and

$$
B(x, t) \supset B\left(y, \frac{t}{a}-d(x, y)\right), t>a d(x, y) .
$$

Then

$$
\frac{\mu B(x, r t)}{\mu B(x, t)} \leq \frac{\mu B(y, a r t+a d(x, y))}{\mu B\left(y, \frac{t}{a}-d(x, y)\right)} \quad \text { for } \quad t>a d(x, y)
$$


and consequently

$$
\varlimsup_{t \rightarrow \infty} \frac{\mu B(x, r t)}{\mu B(x, t)} \leq \varlimsup_{\tau \rightarrow \infty} \frac{\mu B\left(y, a^{2} r \tau+k\right)}{\mu B(y, \tau)}, \quad \tau=\frac{t}{a}-d(x, y),
$$

where $k=a(a r+1) d(x, y)$. For every $r>0$ and an arbitrary positive number $\varepsilon>0$ we have $a^{2} r \tau+k<a^{2} r(1+\varepsilon) \tau$ for large $\tau>\frac{r a+1}{\operatorname{ar\varepsilon }} d(x, y)$. Therefore

$$
\varlimsup_{t \rightarrow \infty} \frac{\mu B(x, r t)}{\mu B(x, t)} \leq \varlimsup_{\tau \rightarrow \infty} \frac{\mu B(y,(r+\varepsilon) \tau)}{\mu B(y, \tau)}
$$

that is,

$$
\mathcal{M}(x, r) \leq \mathcal{M}\left(y, a^{2} r(1+\varepsilon)\right)
$$

Hence

$\lim _{r \rightarrow \infty} \frac{\ln \mathcal{M}_{\infty}(x, r)}{\ln r} \leq \lim _{r \rightarrow \infty} \frac{\ln \mathcal{M}_{\infty}\left(y, a^{2} r(1+\varepsilon)\right)}{\ln r}=\lim _{r \rightarrow \infty} \frac{\ln \mathcal{M}_{\infty}(y, r)}{\ln r-\ln \left(a^{2}(1+\varepsilon)\right)}$,

whence

$$
\lim _{r \rightarrow \infty} \frac{\ln \mathcal{M}_{\infty}(x, r)}{\ln r} \leq \lim _{r \rightarrow \infty} \frac{\ln \mathcal{M}_{\infty}(y, r)}{\ln r}
$$

and then $\lim _{r \rightarrow \infty} \frac{\ln \mathcal{M}_{\infty}(x, r)}{\ln r}=\lim _{r \rightarrow \infty} \frac{\ln \mathcal{M}_{\infty}(y, r)}{\ln r}$ by the arbitrariness of $x$ and $y$. Similarly, the coincidence of the limits as $r \rightarrow 0$ is proved.

In the case where $a=1$ and the measures $\mu B(x, r)$ are continuous in $r$, the last statement of the lemma follows from (4.22).

We dwell on an extension of Theorem 4.3 to unbounded spaces $X$ for the case of inequality (4.17). To this end, we introduce one more characteristic of the space $X$ at infinity:

$$
\underline{\operatorname{dim}}_{X}^{\prime}(\infty)=\sup _{0<r<1} \frac{\ln \left(\varlimsup_{t \rightarrow \infty} \operatorname{esssup}_{x \in \Omega} \frac{\mu B(x, r t)}{\mu B(x, t)}\right)}{\ln r}
$$

Since $\varlimsup_{t \rightarrow \infty} \operatorname{esssup}_{x \in \Omega} \geq \underset{x \in \Omega}{\operatorname{ess} \sup } \varlimsup_{t \rightarrow \infty}$, we have

$$
\underline{\operatorname{dim}}_{X}^{\prime}(\infty) \leq \underline{\operatorname{dim}}_{X}(\infty) .
$$

For many "good" metric measure spaces one has

$$
\underline{\operatorname{dim}}_{X}^{\prime}(\infty)=\underline{\operatorname{dim}}_{X}(\infty)
$$


for instance, for spaces with the measures of balls $\mu B(x, r)$ behaving as $r \rightarrow \infty$ similarly to functions $w(x, r)$ in Lemmas 2.7 and 2.8 as $r \rightarrow 0$.

We also introduce the indices for functions $w$ on an infinite interval, similarly to (2.5)-(2.8), (2.10); for simplicity, in this case we take functions $w$ not depending on a parameter $x \in X$ :

$$
\begin{aligned}
& m_{\infty}(w)=\sup _{r>1} \frac{\ln \left(\lim _{t \rightarrow \infty} \frac{w(r t)}{w(t)}\right)}{\ln r}=\lim _{r \rightarrow 0} \frac{\ln \left(\varlimsup_{t \rightarrow \infty} \frac{w(r t)}{w(t)}\right)}{\ln r}, \\
& M_{\infty}(w)=\inf _{r>1} \frac{\ln \left(\varlimsup_{t \rightarrow \infty} \frac{w(r t)}{w(t)}\right)}{\ln r}=\lim _{r \rightarrow \infty} \frac{\ln \left(\varlimsup_{t \rightarrow \infty} \frac{w(r t)}{w(t)}\right)}{\ln r} .
\end{aligned}
$$

The following theorem provides sufficient conditions for the validity of inequality (4.17), in terms of the indices (they are necessary in the case where $v(r)$ is a power function).

Theorem 4.5. Let $v \in \widetilde{\mathcal{W}}([0, \infty))$. Under assumptions $i)$-iii), the conditions

$$
M(v)<\underline{\mathfrak{d i m}}(X) \quad \text { and } \quad M_{\infty}(v)<\underline{\operatorname{dim}}_{X}^{\prime}(\infty),
$$

are sufficient for the validity of (4.17).

Proof. By Theorem 4.3 and the first condition in (4.26), inequality (4.17) holds for any finite interval $0<r<N$. To guarantee that the constant $C$ in (4.17) does not depend on $N$, we have to use properties of $v(r)$ and $\mu B(x, r)$ at infinity. We may consider the values $r \geq 2$. It suffices to prove that

$$
\int_{1}^{r} \frac{\mu B(x, t)}{t v(t)} d t \leq C \frac{\mu B(x, r)}{v(r)}
$$

as $r \rightarrow \infty$. With the change of variables $t \rightarrow \frac{1}{t}, \rho=\frac{1}{r}$, inequality (4.27) takes the form

$$
\int_{r}^{1} \frac{\mu B\left(x, \frac{1}{t}\right)}{t v\left(\frac{1}{t}\right)} d t \leq C \frac{\mu B\left(x, \frac{1}{r}\right)}{v\left(\frac{1}{r}\right)} .
$$

By Theorem 3.2, the validity of the latter inequality with the uniform constant $C$ not depending on $x$ and $r$, is equivalent to the numerical inequality $M(w)<0$ for the index (2.11) of the function $w(x, r)=\frac{\mu B\left(x, \frac{1}{r}\right)}{v\left(\frac{1}{r}\right)}$. 
After easy calculations, this numerical inequality takes the form

$$
\inf _{r>1} \frac{\ln \left(\varlimsup_{t \rightarrow \infty} \operatorname{ess~sup}_{x \in \Omega} \frac{\mu B\left(x, \frac{t}{r}\right)}{\mu B(x, t)} \frac{v(t)}{v\left(\frac{t}{r}\right)}\right)}{\ln r}<0,
$$

this being a necessary and sufficient condition for the validity of (4.27). Since $\varlimsup_{t \rightarrow \infty} f(t) g(t) \leq \varlimsup_{t \rightarrow \infty} f(t) \varlimsup_{t \rightarrow \infty} g(t)$, we obtain that the inequality

$$
\inf _{r>1} \frac{\ln \left(\varlimsup_{t \rightarrow \infty} \operatorname{ess~sup} \frac{\mu B\left(x, \frac{t}{r}\right)}{\mu B(x, t)}\right)}{\ln r}+M_{\infty}(v)<0,
$$

is sufficient for the validity of (4.28). It is easy to see that the last inequality is nothing else but the inequality $M_{\infty}(v)<\underline{\operatorname{dim}}_{X}^{\prime}(\infty)$, which holds by the assumption.

Acknowledgments. This work was supported by Research Grant SFRH/BPD/34258/2006, FCT, Portugal, and the INTAS project "Variable Exponent Analysis", Nr.06-1000017-8792.

\section{References}

[1] N.K. Bary and S.B. Stechkin, Best approximations and differential properties of two conjugate functions (in Russian), Proceedings of Moscow Math. Soc., 5 (1956), 483-522.

[2] L. Diening, P. Hästö, and A. Nekvinda, Open problems in variable exponent Lebesgue and Sobolev spaces, In: "Function Spaces, Differential Operators and Nonlinear Analysis", Proceedings of the Conference held in Milovy, Bohemian-Moravian Uplands, May 28 June 2, 2004, pages 38-58. Math. Inst. Acad. Sci. Czech Republick, Praha, 2005.

[3] K. Falconer. Techniques in Fractal Geometry, John Wiley \& Sons Ltd., Chichester, 1997.

[4] I. Genebashvili, A. Gogatishvili, V. Kokilashvili, and M. Krbec, Weight Theory for Integral Transforms on Spaces of Homogeneous Type, Pitman Monographs and Surveys, Pure and Applied mathematics, Longman Scientific and Technical, 1998.

[5] A.I. Guseinov and H.Sh. Mukhtarov, Introduction to the Theory of Nonlinear Singular Integral Equations (in Russian), Moscow, Nauka, 1980. 
[6] P. Harjulehto, P. Hästö, and V. Latvala, Sobolev embeddings in metric measure spaces with variable dimension, Math. Z., 254 (2006), 591-609.

[7] P. Harjulehto, P. Hästö, and M. Pere, Variable exponent Lebesgue spaces on metric spaces: the Hardy-Littlewood maximal operator, Real Anal. Exchange, 30 (2004), 87-104.

[8] J. Heinonen, Lectures on Analysis on Metric Spaces, Universitext. Springer-Verlag, New York, 2001.

[9] N.K. Karapetyants and A.I. Ginsburg, Fractional integrals and singular integrals in the Hölder classes of variable order, Integral Transform. Spec. Funct., 2 (1994), 91-106.

[10] N.K. Karapetyants and N.G. Samko, Weighted theorems on fractional integrals in the generalized Hölder spaces via indices $m_{\omega}$ and $M_{\omega}$, Fract. Calc. Appl. Anal., 7 (2004), 437-458.

[11] V. Kokilashvili, On a progress in the theory of integral operators in weighted Banach function spaces, In: "Function Spaces, Differential Operators and Nonlinear Analysis", Proceedings of the Conference held in Milovy, Bohemian-Moravian Uplands, May 28 - June 2, 2004, pages 152-175. Math. Inst. Acad. Sci. Czech Republick, Praha, 2005.

[12] V. Kokilashvili and S. Samko, The maximal operator in weighted variable spaces on metric spaces, Proc. Razmadze Math. Inst., 144 (2007), 137-144.

[13] S.G. Krein, Yu.I. Petunin, and E.M. Semenov, Interpolation of Linear Operators, Volume 54, Translations of Mathematical Monographs, American Mathematical Society, Providence, R.I., 1982.

[14] L. Maligranda. Indices and interpolation, Dissertationes Math. (Rozprawy Mat.), 234 (1985), 49 pp.

[15] V.S. Rabinovich, N.G. Samko, and S.G Samko, Local Fredholm spectrums and Fredholm properties of singular integral operators on Carleson curves acting on weighted Hölder spaces, Integr. Equat. Oper. Theory, 56 (2006), 257-283.

[16] B. Ross and S.G. Samko, Fractional integration operator of variable order in the Hölder spaces $H^{\lambda(x)}$, Internat. J. Math. Math. Sci., 18 (1995), 777-788.

[17] N.G. Samko, Criterion for the Fredholmness of singular operators with piecewise continuous coefficients in the generalized Hölder spaces with weight, page 363. Proceedings of IWOTA 2000, Setembro 1215, Faro, Portugal, Birkhäuser, In: "Operator Theory: Advances and Applications", v. 142. 
[18] N.G. Samko, Singular integral operators in weighted spaces with generalized Hölder condition, Proc. A. Razmadze Math. Inst., 120 (1999), 107-134.

[19] N.G. Samko, On compactness of integral operators with a generalized weak singularity in weighted spaces of continuous functions with a given continuity modulus, Proc. A. Razmadze Math. Inst., 136 (2004), 91113.

[20] N.G. Samko, On non-equilibrated almost monotonic functions of the Zygmund-Bary-Stechkin class, Real Anal. Exch., 30 (2004/2005), 727. Errata: Real Anal. Exchange, 32 (2006/2007), 1-2.

[21] N.G. Samko, Singular integral operators with Carleman shift and discontinuous coefficients in the spaces $H_{0}^{\omega}(\Gamma, \rho)$, Integr. Equat. Operator Theory, 51 (2005), 417-433.

[22] N.G. Samko, Singular integral operator in weighted spaces of continuous functions with non-equilibrated continuity modulus, Math. Nachr., 279 (2006), 1359-1375.

[23] N.G. Samko, Singular integral operators in weighted spaces of continuous functions with an oscillating continuity modulus and oscillating weights, Operator Theory: Advances and Applications, Birkhäuser, Proc. of the conference IWOTA, Newcastle, July 2004, 171 (2006), 323-347.

[24] N.G. Samko, Singular integral operators in weighted spaces of continuous functions with non-equilibrated continuity modulus, Math. Nachr., 279 (2006), 1359-1375.

[25] S.G. Samko, On a progress in the theory of Lebesgue spaces with variable exponent: maximal and singular operators, Integr. Transf. and Spec. Funct., 16 (2005), 461-482.

[26] S.G. Samko, A.A. Kilbas, and O.I. Marichev, Fractional Integrals and Derivatives. Theory and Applications. London-New-York: Gordon \& Breach. Sci. Publ., (Russian edition - Fractional Integrals and Derivatives and some of their Applications, Minsk: Nauka i Tekhnika, 1987.), 1993.

[27] S.G. Samko and Kh.M. Murdaev, Weighted Zygmund estimates for fractional differentiation and integration, and their applications, Trudy Matem. Inst. Steklov, 180 (1987), 197-198. translated in Proc. Steklov Inst. Math., AMS (1989), 233-235.

[28] B.G. Vakulov, Spherical operators of potential type in weighted Hölder spaces of variable order (in Russian), Vladikavkaz. Mat. Zh., 7 (2005), $26-40$. 
[29] B.G. Vakulov, Spherical potentials in weighted Hölder spaces of variable order (in Russian), Dokl. Akad. Nauk, 400 (2005), 7-10.

[30] B.G. Vakulov, Spherical potentials of complex order in the variable order Hölder spaces, Integral Transforms Spec. Funct., 16 (2005), 489497.

[31] B.G. Vakulov, Complex-order spherical potentials in the spaces of variable generalized Hölder order (in Russian). Dokl. Akad. Nauk, 407 (2006), 12-15, translated in Doklady Mathematics 73 (2006), 165-168.

[32] B.G. Vakulov, Spherical convolution operators in Hölder spaces of variable order, Math. Notes, 80 (2006), 683-695.

Universidade do Algarve

Campus de Gambelas

Faro 8005-139

Portugal

(E-mail : nsamko@ualg.pt)

(Received : December 2007) 


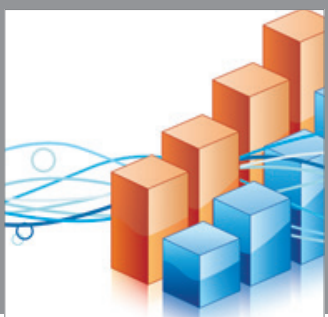

Advances in

Operations Research

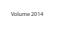

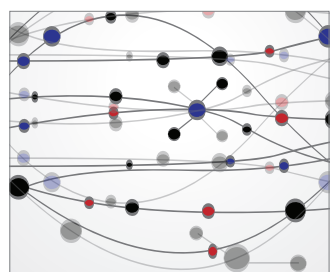

\section{The Scientific} World Journal
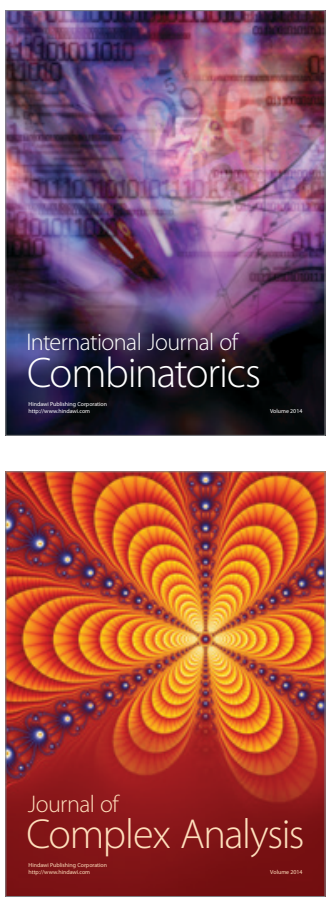

International Journal of

Mathematics and

Mathematical

Sciences
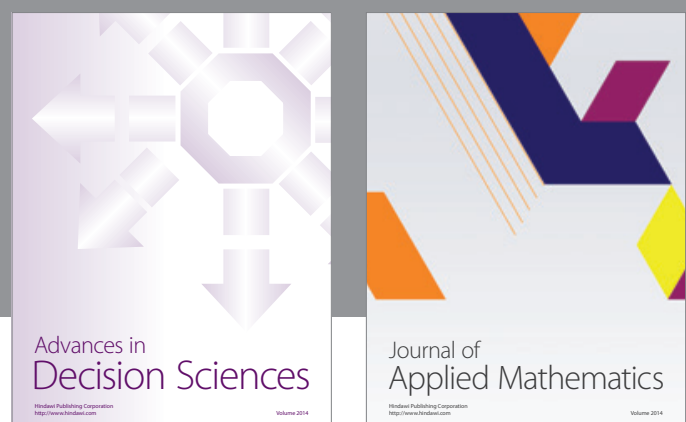

Journal of

Applied Mathematics
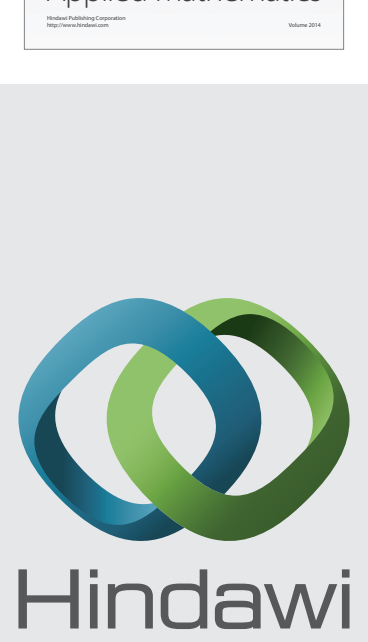

Submit your manuscripts at http://www.hindawi.com
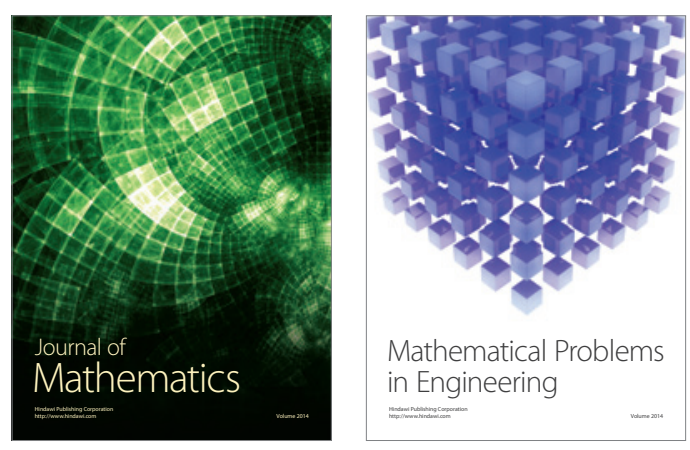

Mathematical Problems in Engineering
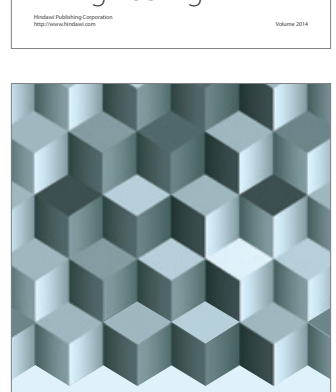

Journal of

Function Spaces
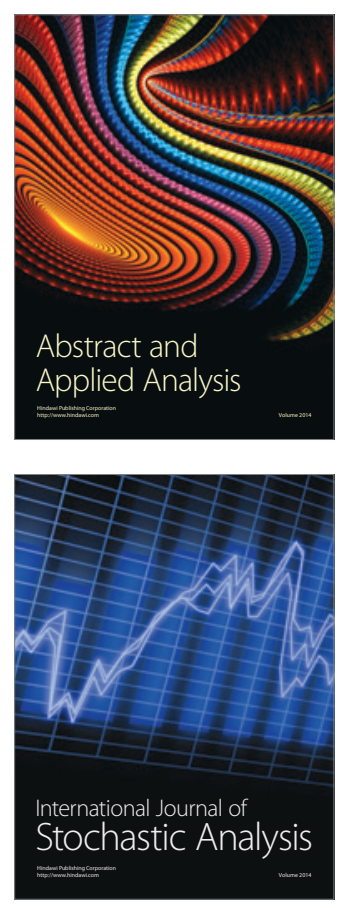

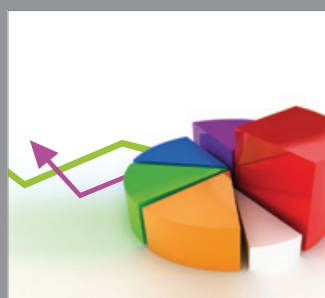

ournal of

Probability and Statistics

Promensencen
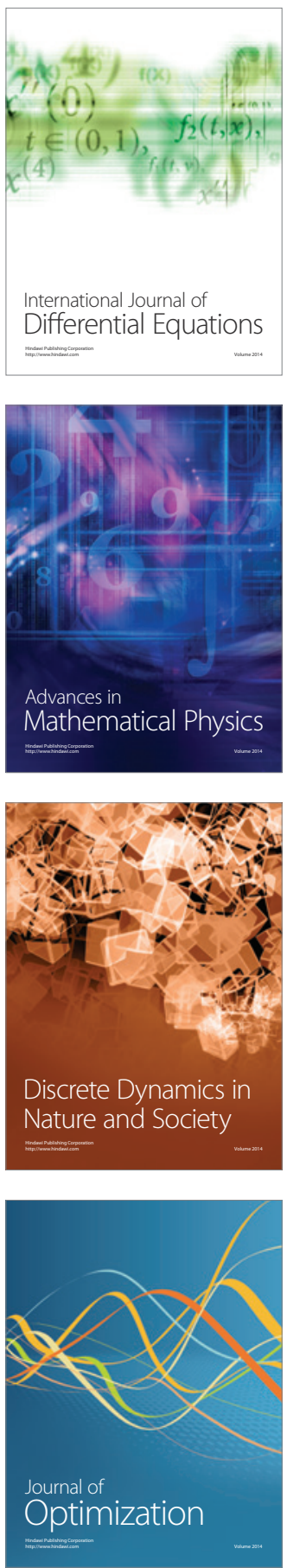